Pedagogía y Saberes n. ${ }^{\circ} 55$

Universidad Pedagógica Nacional

Facultad de Educación. 2021. pp. 55-64

\title{
Emancipación y regulación. Tensión básica en la comunicación-educación latinoamericana*
}

\section{Emancipation and Regulation. Basic Tension in Latin-American Communication-Education Emancipação e regulação. Tensão básica na comunicação-educação latino-americana}

Gilberto Eduardo Gutiérrez**

Para citar este artículo

Gutiérrez, G. (2021). Emancipación y regulación. Tensión básica en la comunicación-educación latinoamericana. Pedagogía y Saberes, (55). https://doi.org/10.17227/pys.num55-11316

* Artículo de reflexión derivado de la tesis doctoral "Sentidos comunes. Saberes y experiencias de maestros y maestras en el campo comunicación-educación en Bogotá", realizada en el Doctorado Interinstitucional en Educación de la Universidad Distrital Francisco José de Caldas.

** Profesor del Departamento de Comunicación de la Pontificia Universidad Javeriana y estudiante del Doctorado Interinstitucional en Educación DIE UD. Orcid: https://orcid.org/0000-0002-5116-676X. Correo electrónico: gilberto.gutierrez@javeriana.edu.co 


\section{Resumen}

Este artículo de reflexión es resultado de la tesis doctoral "Sentidos comunes. Saberes y experiencias de maestros y maestras en el campo comunicación-educación en Bogotá". Mediante una exploración desde América Latina de la relación comunicación-educación es posible reconocer que en la genealogía de sus saberes y prácticas existe una marca profunda de la tensión entre emancipación y regulación, la cual a la vez constituye una comprensión de la lucha dialéctica entre horizontes y proyectos formativos y las formas de la construcción de sentidos en la sociedad. Desde una epistemología del sur y en diálogo entre Freire y Bourdieu, quienes asumen concepciones opuestas acerca de la formación y el sentido dentro de la sociedad, este artículo ratifica a la comunicación-educación como ámbito estratégico de la acción política, la liberación y la resistencia en América Latina.

\section{Palabras clave}

comunicación educación; epistemología del Sur; conocimiento; emancipación; regulación

\section{Abstract}

This reflection article is the result of the doctoral thesis "Common senses. Knowledge and experiences of teachers in the communication-education field in Bogotá". By an exploration of the relation of communication-education, it is possible to identify in the genealogy of knowledge(s) and practices, a tension between emancipation and regulation in Latin America. It constitutes an understanding of the dialectic struggle between formative horizons and projects. From an epistemology of the south and with a dialogue between Freire and Bourdieu that shows their opposite comprehension of formation and sense in society, this paper ratifies communication-education as a strategic field of political action, liberation, and resistance in Latin America.

\section{Keywords}

communication-education; south epistemologies; knowledge; emancipation; regulation

\section{Resumo}

Este artigo de reflexão é o resultado da tese de doutoramento intitulada: "Sensos Comuns: conhecimentos e experiências de professores na área de comunicação-educação em Bogotá". A partir de um viés exploratório, desde a América Latina, da relação entre comunicação e educação, é possível reconhecer que na genealogia desses saberes e práticas existe uma marca profunda da tensão entre emancipação e regulação, e ao mesmo tempo constitui uma compreensão da luta dialética entre horizontes e projetos de formação, bem como as formas de construção de sentidos na sociedade. Desde uma epistemologia do sul, e em diálogo com Freire e Bourdieu, autores que assumem concepções opostas sobre a formação e o sentido dentro da sociedade, o artigo ratifica a comunicação e educação como âmbito estratégico da ação política, da liberação e da resistência na América Latina.

\section{Palavras-chave}




\section{Para localizarse en el mapa}

En Colombia recientemente ha venido desarrollándose una nueva apertura a los debates acerca del campo comunicación-educación. En ellos se establece que en las últimas décadas se ha dado una revoltura (Cubides y Valderrama, 2020) que supera las fronteras de la escuela y los medios, y se encamina al territorio de los movimientos sociales; una redefinición de sus fronteras para pensar desde la cultura, lo popular y el común (Muñoz y Mora, 2016), lo que requiere una revisita para explorar las posibilidades emergentes ante el estallido del campo (Amador y Muñoz, 2018).

Esta apertura de debates que miran la trayectoria de configuración del campo comunicación-educación invita a recuperar las tradiciones invisibilizadas, por ejemplo la de la educación popular y la comunicación alternativa como elementos presentes en la raíz de sus preguntas y sus prácticas, así como a tomar distancia de las perspectivas dominantes que han reducido la comunicación a los medios y la educación a la escuela, empequeñeciendo el territorio de conexiones e interrogantes del campo como lo había sugerido hace ya dos décadas Jorge Huergo (1999) en su balance panorámico.

Una manera de entrar en esta discusión es ir hacia el pasado y recoger algunas de las raíces constitutivas del campo comunicación-educación, a partir de la exploración de la tensión que subyace como interrogante al poner juntas las dos categorías para nombrar luchas y procesos que tienen dificultad en ser nombrados al ver cada una de ellas, comunicación y educación, de manera aislada.

Para tal fin, en este artículo se parte de la discusión sobre las raíces de la relación comunicación-educación pensada desde la epistemología del Sur tomando como base la comprensión de Boaventura de Sousa Santos (2009), luego se abordan algunas claves derivadas de la perspectiva de Bourdieu (1990) y se propone un diálogo con la mirada de Freire en la Pedagogía del oprimido (Freire, s. f.), para finalmente señalar una entrada a la comunicación-educación en busca de que dicha propuesta ayude a dar pistas para la genealogía del campo de saber (no solo académico) de la comunicación-educación.

\section{Comunicación-educación desde una epistemología del Sur}

En Crítica de la razón indolente (2014), Boaventura de Sousa Santos plantea que el paradigma de la modernidad se funda en la tensión dialéctica entre regulación social y emancipación social. La ciencia, en cuanto forma elaborada de la racionalidad moderna, al incorporarse como fuerza productiva dentro de las dinámicas del capitalismo hace que las fuerzas de emancipación sean absorbidas por las de la regulación. Y a la vez, esta forma de producción de conocimiento tiene impacto: "en las luchas sociales emancipatorias, las cuales, una vez prisioneras de la racionalidad científica moderna, acabaron por perder de vista sus objetivos y se transformaron perversamente en nuevas formas de regulación social" (Santos, 2009, p. 63).

De cierto modo, nos encontramos ante formas de la comunicación emancipadora, la educación reguladora, la comunicación reguladora y la educación emancipadora que podrían ser fácilmente identificadas en prácticas y coyunturas concretas a lo largo de la historia. Estas combinaciones expresan la tensión entre regulación y emancipación, y la muestran en prácticas y procesos educativos y comunicativos.

Siguiendo a Boaventura de Sousa Santos, la tensión entre regulación y emancipación fue traducida epistemológicamente en la dualidad entre formas de conocimiento-emancipación y formas de conocimiento-regulación. El autor explica:

el conocimiento-emancipación implica una trayectoria entre un estado de ignorancia al que llamo colonialismo y un estado de conocimiento al que llamo solidaridad. El conocimiento regulación implica una trayectoria entre un estado de ignorancia al que llamo caos y un estado de conocimiento al que llamo orden. (Santos, 2009, p. 63)

Entonces: educación y comunicación son una muestra de la tensión entre regulación y emancipación, y a la vez son objeto de regulación, producto del avance del conocimiento científico. En tanto la educación y la comunicación se van consolidando como campos autónomos y especializados de la acción humana en la modernidad; ellos pueden ser examinados como derivados de la tensión entre regulación y emancipación, y como herederos de esta lucha dialéctica, como se ratifica desde diversas miradas, entre ellas la de Armand Mattelart (1995) para la comunicación, o la de Bourdieu y Passeron (2001) para la educación.

Bajo esta perspectiva, al paradigma de la modernidad concurren diversos fenómenos, entre ellos la educación y la comunicación, materializadas en "artefactos" como la escuela y la prensa; así como en las disciplinas, es decir, la pedagogía, las ciencias de la educación, la comunicología y las ciencias de la comunicación.

De modo que, si asumimos esta perspectiva para comprender el saber en comunicación y educación en América Latina, reconocemos que el campo emergente de comunicación-educación se ha configurado 
históricamente como lucha entre emancipaciones y regulaciones atravesadas por conocimiento-regulación y conocimiento-emancipación, de los cuales el campo comunicación-educación sería su expresión.

A la vez, en América Latina, al vivir la inserción en una modernidad periférica y padecer la imposición de unos modos de ser de las metrópolis, y, por tanto, de la ciencia venida de fuera, con su carácter regulador y colonial, el conocimiento propio emerge bajo el horizonte regulador y se construye como ejercicio anticolonial y emancipador.

Abordar lo educativo y lo comunicativo desde el conocimiento emancipador o confrontar los modos de conocimiento regulador del dominador implica tanto confrontar las imposiciones de la modernidad, como elaborar la propia emancipación. Esto denota el nudo epistemológico en el que se encuentran tanto los procesos de comunicación y educación, como la expansión de sus elaboraciones como conocimiento emancipador o regulador.

La dominación podría expresarse en el modo de imposición de la regulación de la práctica educativa (emancipadora o reguladora), desde el poder de la pedagogía en cuanto conocimiento-regulación. Si el estado no deseado es el de la ignorancia, la educación en su forma de conocimiento-regulación dispone el horizonte del orden en el que se consigue el estado deseado desde la dominación. Pero, a su vez, el orden no es otro que la condición de ignorancia colonialista que debería ser el punto de partida para hallar una trayectoria de conocimiento-emancipación en camino hacia la solidaridad. Esta sería una clave para enfrentar a la educación bancaria y la opresión, siguiendo la perspectiva de Paulo Freire (s. f.), ya que: "en la medida en que el liderazgo niega la praxis verdadera a los oprimidos, consecuentemente, su praxis pierde sentido. Tiende, de esta forma, a imponer su palabra a ellos, volviéndola así una palabra falsa, de carácter dominador" (Freire, s. f., p. 165).

La contradicción para el colonizador es que la alfabetización, es decir, la dotación del logos de la lengua dominante es a su vez el inicio de la conciencia de la dominación y, con ello, el germen de la búsqueda de emancipación en la que es precisamente la transformación misma de la relación entre educadores y educandos lo que da lugar a la emancipación, y ese vector liberador ocurre en la palabra; es decir, en la comunicación como lugar para el tránsito del colonialismo a la solidaridad.

Si no lo hemos hecho mal, lo que expone este primer punto de la perspectiva reflexiva desde la epistemología del sur como lugar para pensar la comunicación-educación es que, en la doble tensión entre regulación y emancipación (y entre el conocimiento-emancipación y el conocimiento-regulación), educación y comunicación también aparecen tensionadas en cuanto fuerzas en pugna en las que se desarrolla la lucha entre sentidos y proyectos formativos que se orientan hacia mundos posibles distintos, vectores que pueden cargarse con el valor regulador o emancipador. Es a esto que hemos llamado el nudo epistemológico como una tensión generadora permanente de luchas entre múltiples fuerzas de emancipación y múltiples fuerzas de regulación.

Se trata de una conexión múltiple entre contexto, comunicación-educación y regulación-emancipación, donde las tensiones mutuas entre los componentes dan lugar a vectores de fuerzas en tensión permanente. Factor que tiene que ver especialmente con reconocer que, por ejemplo, en los ámbitos específicos del maestro y su quehacer se expresa la multiescalaridad en la que se desempeña la comunicación-educación, en tanto el conocimiento-regulación pretende una expansión homogeneizadora, mientras el quehacer en las aulas y en los procesos escolares puede ser ejercicio de la regulación y el control, o puede ser saber de retaguardia, lugar en que la divergencia y la resistencia son posibles.

Atender a la comprensión de la comunicación-educación desde una epistemología del sur significa entonces, en primera instancia, reconocer el sentido de lucha entre regulación y emancipación que subyace a este ámbito como expresión de las tensiones propias de un contexto y época. Y a su vez, ver la comunicación-educación como un ámbito de saber en el que se expresan los conocimientos-regulación y los conocimientos-emancipación, de allí que los maestros como sujetos de saber y de práctica se constituyan en las tensiones que hemos descrito, no solo por la oportunidad de actuar desde y hacia la emancipación o la regulación en su quehacer, como por el hecho de que su propio saber se disputa en el régimen de visibilidad impuesto por las formas de conocimiento-regulación, y ante el reto de validar, hacer reconocible y visible su conocimiento-emancipación.

Esto puede ser expresado en múltiples preguntas concretas, por ejemplo: ¿Qué sucedió entre las últimas décadas del siglo xx y el inicio del xxI para que la epistemología dominante del conocimiento-regulación, expresado en currículos, tecnología educativa e instrumentos técnicos, redujera la posibilidad de la acción de la comunicación-educación a un subsidiario de las formas del conocimiento-regulación, e hiciera casi invisibles las formas de conocimiento emancipador? 0 abrir el horizonte para preguntarse si, tal vez, ¿estaremos aún en el ámbito de la comunicación-educación, 
ante la oportunidad de un tránsito que revalorice el conocimiento-emancipación sobre el conocimiento-regulación, como lo espera De Sousa?

\section{El campo y la reproducción desde Bourdieu}

Para poder asumir a plenitud el planteamiento de la epistemología del Sur como modo de pensar la comunicación-educación, es necesario desmarcarse de la fuerte determinación que ejercen sobre este tema las comprensiones del pensamiento de Pierre Bourdieu, es decir su localización como campo y como espacio propio de la reproducción social. Esto es, tomar distancia del carácter unívoco de la educación al asumir la posibilidad de dejar de soportarla sobre la idea de reproducción y junto con ello repensar la noción de campo para poder ir más allá de los límites establecidos por la epistemología dominante. Como lo plantea Giroux (1992): "la resistencia debe tener una función reveladora, que contenga una crítica de la dominación y ofrezca las oportunidades teóricas para la autorreflexión y la lucha en el interés de la emancipación propia y de la emancipación social" (p. 145).

Para inicios de los años 1970, el trabajo de Bourdieu daba un giro copernicano a la comprensión de la educación, en sus trabajos con Passeron y en especial en La reproducción (2001) ofrecía una perspectiva de ruptura con el modo de pensar establecido hasta el momento. La educación no tiene como fin último la liberación, la ilustración y el pensamiento autónomo, más bien en el hecho de corresponder a una perspectiva de clase, la educación reproduce la desigualdad y ante todo transmite y circula esa ideología a través de las prácticas educativas y su natural violencia simbólica aceptada como hecho propio de la formación de la sociedad desde la perspectiva de la clase dominante.

Si siguiéramos esta perspectiva, el campo de la comunicación-educación podría redefinirse peligrosamente, no como un campo en sí, sino como el modo propio de la eficiencia de la violencia simbólica expandida soportada en los medios de comunicación.

Así como si nos basáramos en esta idea, la comprensión de la comunicación-educación se traduciría de manera limitada como el contacto entre dos campos estructurados e interconectados, el de la comunicación y el de la educación que se ven modificados desde inicios de siglo xx por el impacto que en el primero tiene una transformación radical del habitus, en términos de la expansión y uso direccionado de artefactos que soportan nuevas formas de expansión del poder simbólico dominante.
En este sentido, por ejemplo, los medios de comunicación en manos del estado no solo constituyen un objeto particular de soporte del poder simbólico, sino que son a la vez un poder en sí mismo que en su expansión modifica el habitus y, con ello, se hacen cuerpo en los sujetos. Así se expresa en La reproducción:

Por el hecho de que toda Acción Pedagógica en ejercicio dispone por definición de una Autoridad Pedagógica, los emisores pedagógicos aparecen automáticamente como dignos de transmitir lo que transmiten y, por tanto, quedan autorizados para imponer su recepción y para controlar su inculcación mediante sanciones socialmente aprobadas o garantizadas. (Passeron y Bourdieu, 2001, p. 61)

Es tal vez este poder el que, para ese momento, hace creer que las formas de comunicación en expansión contienen y aparecen como una alternativa acertada para cumplir los fines educativos desde la dominación.

Aunque este fenómeno ya era así anteriormente, desde La reproducción sabemos que una acción pedagógica se hace eficiente en la conformación de un discurso y en una forma de violencia simbólica. La expansión de estos y su uso en el marco de la Guerra Fría para poder establecer procesos de control y dominación en países del tercer mundo, muestra una zona de lucha particular que expande el campo de la educación, mostrando su eficacia simbólica, pero que no da muestras de la emergencia de un campo nuevo. En consecuencia, sirve, de algún modo, con esta comprensión a sostener la dominación.

En este sentido, los nuevos instrumentos de la violencia simbólica ocuparían no solo el habitus del campo cotidiano de la comunicación sino que impactan el campo de la escuela y sus poderes simbólicos bajo la idea de una cierta illusio de la eficiencia por vía de la modificación de lo comunicativo que cumple el doble interés del dominador de reproducir su forma de poder y de construcción de lo común a través de la homogenización de las herramientas comunicativas y a la vez la construcción de la confianza en los medios como capaces de modificar el habitus. Dicho de otro modo, es el proceso por el cual, bajo la posible dominación absoluta, se vuelve más agudo el poder simbólico de la educación dentro y fuera de la escuela para hacer más eficiente la violencia simbólica, por eso la educación arrastra consigo los medios de comunicación y estos se vuelven un escenario propio del poder establecido.

Pero, para dejar alguna oportunidad a lo comunicativo-educativo: ¿Podría ser la comunicación el lugar de la construcción de otro poder simbólico? ¿Podría serlo lo educativo si escapara a las lógicas de la reproducción? ¿Sería posible huir de ellas? Al 
menos desde la perspectiva de la reproducción no. Y en esta perspectiva lo comunicativo aparecería como una expansión unívoca del poder simbólico de la educación y no constituirían un campo nuevo. Es precisamente esta lectura vista desde la práctica, la que, para ese mismo tiempo, estaba siendo puesta en cuestión por vía de hecho por Paulo Freire y por las diversas acciones transformadoras que como acciones liberadoras se expandían en América Latina.

\section{El capital militante y la emancipación como illusio}

Pensar el campo comunicación-educación desde la reproducción haría imposible asumir el que allí se propiciara algo distinto de la expansión de la dominación de clase. Sin embargo, si observamos la presencia de unos modos de hacer, pensar y movilizar persistentes en el habitus de lo comunicativo-educativo como formas de resistencia y reexistencia en América latina, y si a la vez aceptamos que la lucha persiste por el poder del campo en cuanto capital simbólico de saber, y que las confrontaciones le apuestan hoy a un cambio en la lógica del juego, es decir sobre el deseo de liberación como el signo que constituye los saberes de la comunicación-educación, estamos ante una relectura del campo donde la reproducción no es la única vía, sino que la persistencia de la crítica y la constitución de un habitus liberador hablaría de un territorio nuevo al que posiblemente nombraríamos como resistencia o reexistencia y, en términos de Borudieu, en disposición de luchar por el poder en el campo reinventando su juego ${ }^{1}$.

Si basados en la perspectiva de Bourdieu intentáramos reconstruir la estructura del campo de comunicación-educación y con ello poder llevar a cabo el análisis de correspondencias múltiples, tenemos que, a partir de la categoría de espacio social entendido como un "espacio pluridimensional de posiciones donde toda posición actual ( de un agente o grupo de agentes) puede ser definida en función de un sistema pluridimensional de coordenadas, cada una de ellas ligada a la distribución de una especie de capital dife-

1 Para probar esta posibilidad, es necesario pasar de este debate conceptual al terreno y la exploración con metodologías que pudieran describir las luchas del campo a través de las trayectorias de actores colectivos y procesos. También, es pertinente reconstruir las prácticas y discursos propios en ejercicio y con ello ver la posibilidad del habitus liberador que se ha venido constituyendo. A la vez se hace necesario describir tanto la institucionalización del campo en sus modos académicos como en aquellos que no lo son y que demarcarían validaciones de una epistemología que diverge del saber académico. Es posible que este tránsito ayude a configurarlo la construcción de la categoría experiencia y la definición del modo de llegar a recuperarla. rente" (Bourdieu, 1990, p. 283). Deberíamos preguntarnos ¿en qué consistiría entonces el espacio social de los actores (proyectos, procesos, experiencias) de comunicación-educación?

Si en lugar de pensar que la reproducción es la illusio del campo comunicación-educación y, en clave de epistemología del Sur, pudiéramos proponer como illusio a la tensión entre regulación y emancipación, entonces, el ejercicio de reconstruir las correspondencias múltiples hablaría de una distribución de capital en los proyectos, procesos y experiencias con capacidad de transformación y sus rasgos nos mostrarían una pista interesante a partir del alcance, escala, replicabilidad y sobre la participación y grado de implicación e intensidad del trabajo que se realiza desde allí.

Pero tal vez lo más interesante de estas preguntas sería poder ver el conjunto, y en él, identificar dónde están las experiencias en las que puede ser visible un acumulado de capital, y en el poder entender que dicho capital no deriva solamente de la reproducción sino de la transformación. Esta posibilidad parte de asumir el espacio social como lugar de lucha entre dicho nuevo capital y las formas institucionales, sus reglas de reproducción, los objetos privilegiados o dominantes en el campo, etc.

Entonces, lo que se pone en debate en la definición de la comunicación-educación como campo desde Bourdieu es precisamente que aquello que motiva la lucha, la illusio, en la pugna que hemos planteado entre regulación y emancipación nos impone la redefinición del campo en un horizonte político y por tanto es la posibilidad de un capital emancipador lo que mejor definiría dicha apuesta en América latina. Aquí habría que ir más despacio y ver la definición que el mismo Bourdieu hace del capital militante como forma específica de la illusio, es decir, el modo en que se juegan los sentidos de la transformación. Es decir, ¿si el fin de la educación no se restringiera a la reproducción? ¿Y si la lucha también fuera por la emancipación?

Si pudiéramos tener los puntos o zonas en las que el análisis de correspondencias múltiples sugeriría ahondar, estaríamos en condiciones de seleccionar un grupo de experiencias, en las que es viable hacer un abordaje de las trayectorias. Esto implica que un abordaje diferente estaría marcado por la posibilidad de comprender "la serie de posiciones sucesivas ocupadas por un mismo agente (o por un mismo grupo) en un espacio en sí mismo, en movimiento y sometido a incesantes transformaciones" (Bourdieu, 1997, p. 82). En otras palabras, por ejemplo, ver en el relato de sujetos, lideres, miembros participantes, un conjunto de pistas que permiten identificar estadios sucesivos del campo ante los cuales han ocurrido acontecimientos, 
acciones o procesos diversos: crisis, logros y movilizaciones, reconfiguraciones, estados de disolución o momentos y a la vez leer con ellos el modo en que han comprendido el contexto, las tensiones sociales, las demandas institucionales, los cambios tecnológicos y las diversas determinantes de su proceso.

Pero, sobre todo, en la medida en que se busca comprender el modo en que la illusio de transformación y la resistencia o la reexistencia se llevan a cabo será necesario dar cuenta también del capital militante. Idea que no es ajena a la tradición bourdeana y se desarrolla en el texto Dominación y movilizaciones, estudios sociológicos sobre el capital militante y el capital escolar de Franck Popeau (2007).

Este autor sugiere un camino posible de trabajo al identificar el aprendizaje de las organizaciones y el valor de la experiencia en "los aprendizajes conferidos por el militantismo, en las competencias importadas del exterior, así como en las que son 'aprendidas en el taller', en lo que hemos elegido llamar, al menos provisoriamente, capital militante" (p. 39). Este factor se complementa en la búsqueda de definición del capital militante según el cual:

más allá de la manera por la cual los agentes sociales lo adquieren y luego lo usan, es importante aprehender la lógica de las transferencias por las cuales el capital militante (una parte, al menos) puede ser utilizado en otros espacios e, inversamente, el modo por el cual ciertas propiedades eficientes en otros dominios pueden ser transferidas en esas instancias. (Popueau, 2007, p. 44)

El capital militante resulta entonces un factor central en la comprensión de lo que los proyectos, procesos y experiencias de comunicación-educación llevan a cabo, pero su matiz más interesante está en que nos pone en el rumbo de reconocer no solo el saber del hacer sino el sentido y la illusio que subyace a esta acción. Es por esto, por lo que para el caso de la resistencia y al reexistencia que definimos en el segundo acápite de este texto como el centro de la tensión comunicación-educación, es posible proponer la exploración de un capital que podemos concebir, al menos, como derivado del anterior y que se podría describir como el capital emancipador. Este daría cuenta no solamente de la disposición a articularse y promover acciones políticas orientadas a un fin y vincularse con ellas, sino que estaría atento al hecho de que dicho capital ofrece la posibilidad de reconocer en el relato los proyectos y deseos de transformación convertidos en fuerzas en pugna y en maneras de recorrer el campo. Dicho de otro modo, ver la carga de sentido que enmarca e indica una posición en el espacio social.
Tomando en cuenta que metodológicamente las trayectorias serán un elemento determinante en el proceso de comprensión de las experiencias procesos y proyectos de comunicación-educación emancipadores y por tanto en la comprensión del capital emancipador. La trayectoria entendida como puesta en juego de un capital emancipador en el curso de las contradicciones en las que se juega, los cambios de dirección y el riesgo de perder rumbos en la apuesta de ese capital emancipador, a veces cooptado a veces encubierto, podrían dar cuenta de la forma de la lucha por los capitales en este campo ${ }^{2}$. Probablemente estos relatos de pugnas y avatares, la manera en que hacen cuerpo la estructura de tensiones del espacio social actual, pueda darnos pistas para saber si podemos mantener la esperanza emancipadora o si aquello que resta de emancipación puede simplemente colocarse como un rastro más de la dominación, pero sobre todo si aún desde la comunicación-educación restan opciones de construir lo común y hacer-lo posible.

Esta lectura posicionada en la epistemología del Sur enfrenta la tensión entre regulación y emancipación poniendo en el centro y primer plano a la illusio de liberar o controlar en la expresión capital emancipador. Dejando en claro que a la tensión comunicación-educación subyace la presencia de la contradicción, de la lucha dialéctica entre regulación y emancipación.

\section{Comunicación educación estalla los límites del campo: repensar el habitus en clave de Freire}

Bajo esta mirada lo que constituye el objeto de la lucha, de uno y otro lado y con signos contrarios, es precisamente el deseo transformador de ambos, surgido de la coyuntura en la que nace este saber, es decir la de los proyectos/procesos de liberación resistencia/reexistencia surgidos en América Latina desde los albores de los años 1950 hasta la actualidad, y de los proyectos de contención direccionamiento de este mismo deseo transformador en la configuración de las sociedades latinoamericanas de la segunda mitad de siglo xx como opresión/control/dominación. Hipotéticamente se podría establecer que el capital en juego es el capital transformador. Con estas pistas si seguimos la idea de Bourdieu podríamos hallar lo que está en juego y con ello es más viable mirar hacia la configuración de campo y habitus.

2 Por supuesto, esta relación posee una profunda y sugestiva conexión con la comprensión de Michel de Certeau que para los años 1980 pondrá en su Invención de lo cotidiano (2000, p. L) la distinción entre tácticas y estrategias hallando las maneras de hacer como éxitos del débil contra el más fuerte. 
Si entendemos el campo como la historia hecha cosas, podríamos indicar que esta doble condición del campo se expresa de manera contradictoria: de un lado, en la institucionalización de lo comunicación-educación en grupos de investigación, universidades y programas de formación, eventos académicos, publicaciones y figuras de expertos, y de otro, como proyectos alternativos, trayectorias difusas, sabedores y procesos emergentes e intuitivos de resistencia, actuados sin una estructuración de tipo académico definido, pero en gran parte capaces de poblar zonas diversas de los social para divergir y crear otros modos de hacer educación y comunicación expandiendo un sentido emancipador y crítico. 0 , en términos de Santos, el conocimiento-regulación en tensión con el conocimiento-emancipación.

A la vez, en la perspectiva del habitus, en tanto lo social incorporado, es posible ver cómo los proyectos de comunicación-educación dominante se expresan en protocolos, rutinas de acción, modelos de trabajo, planes y esquemas reiterados, réplicas y modelos transferibles; en tanto las propuestas de otros saberes se plantean desde la particularidad y proponen rutas divergentes, coyunturales y poco estructuradas, atienden a lo táctico a la oportunidad a la deriva de la vida como lo hemos acotado antes en la clave de De Certeau.

En esta perspectiva, Bourdieu nos deja un amplio campo de preguntas: ¿Cómo ver la illusio cuando el mismo interés puede cargarse de sentidos contradictorios según la apuesta de las fuerzas en lucha? ¿Puede un campo ser a la vez sólido y difuso y de qué modo se puede nombrar la zona marginal que no solo se opone, sino que se funda en una epistemología diferente que busca replantear el juego mismo y sus reglas, o es simplemente una forma concreta de campo en tanto estado de la lucha por el capital simbólico? ¿Son estos que vemos como difusos y no estructurados modos de saber que se corresponden con los herejes de los que habla el autor? (Bourdieu, 1990, p. 138). ¿Puede vivir un saber en el margen y hacerse como un poder en el margen sin querer rehacer el campo ni establecer su hegemonía en el campo existente? ¿Debería interpretarse la transformación con sentido liberador como una forma nueva de hegemonía en el campo hecha de este otro tipo de saber?

Es decir, si lo que varía no es la lucha sino el hecho mismo de que la lucha en perspectiva de oposición sea la salida: creo que esta es la lección freireana menos leída en la Pedagogía del oprimido, se trata de la lucha para acabar con la opresión como forma de relación humana y no para pretender ser ahora un nuevo opresor. Ese desplazamiento epistemológico es el que se debe usar para comprender la configuración del campo y para saber si la idea misma de lucha por un capital es la relación que describe aquello que está a la base de la configuración de todos los saberes.

Este punto debe ser analizado en gran parte en la manera en la cual se va creando un lugar social para este saber y en ello se va construyendo tanto su modo de ser institucional (posgrados, academia, investigaciones, etc.) y su modo de ser de resistencia (prácticas, movimientos y organizaciones), saberes prácticos que no se han figurado en discursos de comunicación-educación aún, pero que lo son en la manera de construir un habitus.

Visto en clave de epistemología del Sur, lo que implica el planteamiento bourdeano es la aproximación de toda configuración de campo a la idea de conocimiento regulador, mientras que esta otra mirada plantearía la posibilidad permanente de mutación de campo y habitus a partir de la acción de un carácter emancipador del saber. Su naturaleza emancipadora es también la acción de redefinición del campo más allá de la epistemología dominante y por tanto el transito esperado por Santos de lo regulador a lo emancipador.

\section{Bourdieu y Freire, desde el Sur: ¿Acaso la resistencia?}

Una coincidencia temporal une a Paulo Freire y Pierre Bourdieu. Hacia 1970 los dos publican obras cruciales que se asemejan en el asunto del que se ocupan: la educación y su manera de sostener el poder y la dominación. Los dos comprenden, en una coyuntura similar, aunque en regiones distantes, el sentido de la educación y de las formas de violencia simbólica que lo soportan. Sin embargo, miran en direcciones diferentes.

El uno comprende el mundo agotado de la posguerra que ha hecho posible el Mayo del 68 y las nuevas perspectivas y movimientos intelectuales y acciones políticas como direccionado a la reproducción y en algún modo hacia la sin salida del poder establecido. El otro, desde las zonas colonizadas del agro brasileño y desde los analfabetismos y las exclusiones de la palabra y del propio reconocimiento de los movimientos populares en América Latina, busca concientizar de algo que no pertenece el campo intelectual ni busca el acceso a la cultura burguesa, sino que se ocupa de validar el sentido propio de los sectores marginados, un modo en el que deben autorreconocerse y con ello superar la dialéctica de la opresión, dando lugar a su mundo sin llegar a ser los dominadores.

Freire es la pista clave para la construcción de proyectos alternativos desde la comunicación-educación, su pedagogía crítica es un referente compartido de los 
proyectos que persisten aún hoy en la idea de emancipación crítica y dialógica, como un modo de actuar y de oponerse y recrear la vida en muchas zonas de América Latina y el mundo. Sin embargo, ese mundo no ha cambiado, o al menos no lo ha hecho en la dirección que la illusio emancipadora sugeriría.

Bourdieu deliberó por años acerca de los cambios necesarios y desde su crítica hizo visibles las tramas internas del poder y de la dominación de clases no solo en la naturalización de su violencia simbólica y su poder, sino en su expansión de formas diversas y en las distintas dimensiones de lo social, con luchas que persisten, aunque sin posibilidad de modificación. Su acumulado crítico propicia la esperanza del develamiento.

Las epistemologías que conducen a uno y otro son diferentes. Bourdieu busca acompasar lo objetivo y lo subjetivo en un constructivismo estructural, su comprensión ve la incidencia mutua entre lo establecido y lo emergente, entre campo y habitus. La de Freire y con ella la de la comunicación-educación parece centrarse en lo particular, en lo no universalizable, y a su vez en lo que desde otros se ha entendido como multiescalaridad y con los saberes de retaguardia; es decir, con aquel universo de lo declaradamente emancipador, no por oposición sino por su acción divergente deliberada, focal. Las perspectivas parecerían irreconciliables, pero no es así.

Comunicación-educación vista en clave de Freire es un lugar en el que se construye con los diversos actores un proceso de reinvención emancipadora desde la palabra, el lenguaje y las múltiples alfabetizaciones. Si pensamos esto en clave bourdeana, podemos observar que en las luchas por el dominio y constitución del campo las fuerzas propias del poder establecido siguen manteniendo las formas hegemónicas de la reproducción, hacen comunicación-educación para la expansión instrumental de la técnica, para la persecución de estándares y para la expansión de las lógicas dominantes en el capitalismo global. La clave está en la disposición de un habitus crítico; es decir, de persistir en el carácter develador del poder en la crítica de Bourdieu.

Por eso, el resurgimiento de movimientos populares, la recuperación de memorias culturales profundas, de pedagogías y formas comunicativas y cosmovisiones ancestrales parecen hablar de la existencia de un nuevo capital simbólico emancipador que se ha incorporado como apuesta política en diversas regiones de América Latina entre quienes se han propuesto "hacer ese mundo otro con sus propias manos, poniendo en juego su imaginación y sus sueños; con modos diferentes de hacer, que no son calco y copia de la sociedad dominante, sino creaciones auténticas, adecuadas al nosotros en movimiento" (Zibechi, 2015, p. 39), los cuales se ponen en juego en el campo. Podría hablarse de que habría una renovada illusio: la posibilidad liberadora y la puesta en juego del capital simbólico emancipador/transformador. Podría verse allí la lucha de otras fuerzas de un campo que construido en el margen y que puede traerse a la vez como oposición al signo del dominador como comunicación-educación para el desarrollo, el control y la manipulación, es decir como el signo de la resistencia y como comunicación-educación liberadora.

Algunas ideas nos ayudarían a confirmar la intuición. En 1998, al lado de Loic Wacquiant, Bourdieu escribe Sobre las astucias de la razón imperialista (2005). Lejos de los radicales puntos de la reproducción y enmarcado en los debates de la coyuntura, los autores proponen una idea contundente: "el imperialismo cultural descansa sobre el poder de universalizar particularismos ligados a una tradición histórica singular, haciéndolos desconocer como tales" (Bourdieu y Wacquiant, 2005, p. 205). Esta idea toca muy cercanamente con lo que se podrá llamar una epistemología abismal en Santos, es decir la idea de que la crítica sobre el soporte epistemológico dominante exige darles lugar a las otras perspectivas. Es decir, que la cita y el texto habla de cómo se expanden las ideas y las categorías de la academia dominante en diversos campos, como el de la comunicación-educación, y a la vez habla de una necesidad crítica que es la que cada vez más claramente los movimientos emergentes en América Latina cobran lugar: superar la perspectiva del dominador, quitándose los lentes que le imponen un único modo de sentir para pensar en las rutinas y las ideas comunes, en la posibilidad del disenso. Tal como lo dejaría ver una comprensión de la acción de la comunicación-educación, la reinvención crítica de la vida ocurre en el habitus.

La mirada de Freire como la construcción de un habitus divergente a partir de la resistencia simbólica marca la pista de los diversos movimientos de resistencia y la superación de la reproducción, una lectura de la manera de privilegiar lo común, y con ello los sentidos construidos en los tejidos comunicativos que desde el margen inventan otras posibilidades. La oportunidad permanente de estallar las fronteras y delimitaciones de cualquier campo. Lo aún en pugna. La doble tensión que redunda en la base de la comunicación-educación, en la pugna entre regulación y emancipación. Las invisibilidades confrontadas por la redefinición del capital emancipador, la ruptura de la frontera de campo desde los saberes y no desde lo disciplinar. Lo posible y no solo la reproducción. 


\section{Referencias}

Amador, J. C. y Muñoz, G. (2018). Comunicación-educación en Abya Yala: lo popular en la reconfiguración del campo. Nomadas, 49, 47-67.

Bourdieu, P. (1990). Espacio social y génesis de las "clases". En Sociología y cultura (pp. 281-309). Grijalbo.

Bourdieu, P. (1997). Razones prácticas, sobre la teoría de la acción. Anagrama.

Bourdieu, P. y Passeron, J.-C. (2001). La reproducción: elementos para una teoría del sistema de enseñanza. Popular.

Bourdieu, P. y Wacquiant, L. (2005). Sobre las astucias de la razón imperialista. En P. Bourdieu y L. Wacquiant, Intelectuales, política y poder. Eudeba.

Cubides, H. y Valderrama, C. (2020). Prácticas de colectivos sociales y revolturas del campo comunicación-educación. En J. C. Amador, S. Rojas y R. Solano (eds.), Comunicación-educación en contextos de globalización, neoliberalismo y resistencia (pp. 19-41). Universidad Distrital Francisco José de Caldas.

De Certeau, M. (2000). La invención de lo cotidiano. Vol. 1. Universidad Iberoamericana.

Freire, P. (s. f.). Pedagogía del oprimido. América Latina.
Giroux, H. (1992). Teoría y resistencia en educación. Una pedagogía de la oposición. Siglo XXI editores.

Huergo, J. (1999). Comunicación-educación: itinerarios transversales. En C. Valderrama (ed.), Comunicación-educación: coordenadas abordajes y travesías (pp. 3-25). DIUC Universidad Central y Siglo del Hombre Editores.

Mattelart, A. (1995). La invención de la comunicación. Siglo XXI Editores.

Muñoz, G. y Mora, A. (2016). Comunicación-educación en la Cultura para América Latina. Uniminuto.

Passeron, J. y Bourdieu, P. (2001). La reproducción. Elementos para una teoría del sistema de enseñanza. Popular.

Popueau, F. (2007). Dominación y movilizaciones. Estudios sociológicos sobre el capital militante y el capital escolar. Ferreyra Editor.

Santos, B. d. (2009). Una epistemología del Sur. Clacso-Siglo XXI Editores.

Santos, B. d. (2014). Crítica de la razón indolente. Contra el desperdicio de la experiencia. Vol 1. Para un nuevo sentido común. Desclee de Brouwer.

Zibechi, R. (2015). Descolonizar el pensamiento critico y las prácticas emancipatorias. Desde Abajo. 